\title{
Discrimination of Radix Polygoni Multiflori from different geographical areas by UPLC-QTOF/MS combined with chemometrics
}

\author{
Jin-Fa Tang ${ }^{1 \dagger}$, Wei-Xia Li ${ }^{1 \dagger}$, Fan Zhang ${ }^{1}$, Yu-Hui Li ${ }^{1}$, Ying-Jie Cao ${ }^{1}$, Ya Zhao ${ }^{1}$, Xue-Lin Li ${ }^{1 *}$ and Zhi-Jie Ma ${ }^{2 *}$
}

\begin{abstract}
Background: Nowadays, Radix Polygoni Multiflori (RPM, Heshouwu in Chinese) from different geographical origins were used in clinic. In order to characterize the chemical profiles of different geographical origins of RPM samples, ultra-high performance liquid chromatography quadrupole time of flight mass spectrometry (UPLC-QTOF/MS) combined with chemometrics (partial least squared discriminant analysis, PLS-DA) method was applied in the present study.

Methods: The chromatography, chemical composition and MS information of RPM samples from 18 geographical origins were acquired and profiled by UPLC-QTOF/MS. The chemical markers contributing the differentiation of RPM samples were observed and characterized by supervised PLS-DA method of chemometrics.

Results: The chemical composition differences of RPM samples derived from 18 different geographical origins were observed. Nine chemical markers were tentatively identified which could be used as specific chemical markers for the differentiation of geographical RPM samples.

Conclusions: UPLC-QTOF/MS method coupled with chemometrics analysis has potential to be used for discriminating different geographical TCMs. Results will help to develop strategies for conservation and utilization of RPM samples.
\end{abstract}

Keywords: UPLC-QTOF/MS, Radix Polygoni Multiflori, Different geographical origins, Chemical markers, Partial least squared discriminant analysis

\section{Background}

Radix Polygoni Multiflori (RPM, Heshouwu in Chinese) is the dried root tuber of Polygonum multiflorum Thunb. (Fam. Polygonaceae). As one of the most popular and precious traditional Chinese medicines (TCMs), it is officially documented in the Chinese Pharmacopoeia for

\footnotetext{
*Correspondence: ydsys507@126.com; lixuelin450000@126.com; 13811647091@163.com

†Jin-Fa Tang and Wei-Xia Li contributed equally to this work

${ }^{1}$ The First Affiliated Hospital of Henan University of Chinese Medicine, No. 19, Renmin Road, Jinshui District, Zhengzhou 450000, People's Republic of China

2 Beijing Friendship Hospital Affiliated to Capital Medical University, No.

95, Yongan Road, Xuanwu District, Beijing 100050, China
}

calming the nerves, nourishing blood, activating channels and collaterals, tonifying liver and kidneys, and preventing the premature graying of hair. Many 1000 years of clinical practice of TCM has demonstrated the effect of RPM in terms of preventing dementia and improving memory [1]. As a traditional medicine and dietary supplement for health, it has also been considered effective in antiaging and increasing longevity $[2,3]$. According to modern researches, RPM has the pharmacological effects of enhancing immunity, anti-atherosclerosis, anti-inflammatory, antibacterial, anti-cancer, anti-mutagenic, antioxidation, increasing DNA repair, and improving adipose metabolism [4-6]. 
With the extensive application of RPM, its safety has drawn widespread attention. More and more literatures showed that RPM and RPM-containing herbal products had the adverse effects of hepatotoxicity [7]. The RPM dose in the Chinese Pharmacopoeia (2005 edition) is 6-12 g [8]. According to the safety considerations, the recommended dose of RPM was adjusted to 3-6 g in the 2010 edition of the Chinese Pharmacopoeia [9]. In addition, the safety, quality and efficacy of RPM samples may vary greatly because of the different geographical origins. As well known, medicinal herbs in authentic producing areas had the best quality, which can produce the best pharmacological effect. The place where authentic medicinal herbs produced is called the "trueborn area". RPM is widely distributed in China's southwest, central, south, east and other regions, including Sichuan, Yunnan, Guizhou, Chongqing, Guangdong, Guangxi, Jiangsu, Anhui, Hubei, Hunan, Henan, Jiangxi, Shanxi, Gansu and other provinces and cities [10]. Owing to its many origins, the "trueborn area" of RPM is still being studied. It is consensus that the effect of Chinese medicine relies on the role of its multi-component. There are large difference among the chemical composition and content of RPMs because of its different species and origins, which will cause a greater impact on its efficacy. Therefore, the distinction among RPM samples from different origins is essential for determining the trueborn area of RPM and for selecting good quality RPM to treat diseases.

The introduction of new analytical techniques and the application of novel data analysis methods have greatly promoted the quality assurance of TCM. From the literatures summary, we found that fingerprinting quality control of RPM from different geographical origins was determined by thin-layer chromatography (TLC) scanning and high-performance liquid chromatography (HPLC) [11, 12]; the quality of various commercial specifications of RPM and its dregs was evaluated by HPLC [13]; the quality control of RPM from different origins was determined by infrared spectrum (IR), inductively coupled plasma-atomic emission spectrometer (ICPAES) and LC-mass spectrometry (MS), etc. [14-16]. However, the authentic correlation between geographical distribution regions and chemical variation in RPMs has been rarely reported.

It was reported that RPM mainly contains anthraquinones, stilbene glycosides, phospholipids, phenols, flavonoids, etc. [17]. The present study is aimed to classify and characterize RPM samples from different geographical origins based on the chemical compounds by chemometrics. Chemometrics is an interdisciplinary science involving mathematics and statistics, chemistry and computer science. In recent years, chemometrics have gained more attention along with the development of computer science. Chemometrics combined with liquid chromatography and other spectrometric methods are widely used in many fields concerning TCMs, such as the comparison of different species [18], quality control and modernization of TCM [19]. Herein, eighteen RPM samples from 10 counties of 4 provinces were analyzed using ultra-high performance liquid chromatography quadrupole time of flight mass spectrometry (UPLC-QTOF/MS). And partial least squared discriminant analysis (PLS-DA) of chemometrics approach was applied to classify different RPM samples and find chemical variables that contribute to the differentiation of RPMs. Furthermore, the Progenesis QI software (v2.0, Waters Corporation, Milford, USA) with fast, objective, and reliable characteristics was used for the chemometrics statistical analysis, which had been already used to found the chemical differences among the different extracts of RPM and RPM Praeparata [20], but it only analyzed the differences between the water and ethanol extracts of RPM and RPM Praeparata. Therefore, it would also be feasible to use this method to find different chemical markers among RPMs from different geographical origins. The results can provide more effective strategy guidance for the utilization and domestication of RPM.

\section{Methods}

The Minimum Standards of Reporting Checklist (Additional file 1) contains details of the experimental design, and statistics, and resources used in this study.

\section{Chemicals and reagents}

Acetonitrile (HPLC grade) and formic acid were purchased from Merck KGaA (Darmstadt, Germany); ultrapure water was purified by a Milli-Q system (Milford, MA, USA).

\section{Plant materials}

Seventeen species of planted or wild RPM samples were collected from 10 counties, 4 provinces of China; and one kind of RPM sample (S13) was purchased from pharmacies (Table 1). All the herbal samples were authenticated by the authors. The corresponding voucher specimens were stored in the laboratory for drug metabolism and pharmacokinetics (DMPK) Research of Herbal Medicines, the First Affiliated Hospital of Henan University of Chinese Medicine.

\section{Sample preparation}

The 18 RPM samples were sliced, dried, and powdered. The powdered samples were screened trough no. 4 sieve, respectively; and $0.25 \mathrm{~g}$ was extracted with $25 \mathrm{~mL} 70 \%$ ethanol for 30 min by reflux extraction method, cooled at room temperature and weighted. The reduced weight 
Table 1 Geographical information of 18 RPM samples

\begin{tabular}{|c|c|c|c|}
\hline No. & Source & Longitude & Latitude \\
\hline S1 & Cultivated in Xinzhou town, Huangping county, Guizhou province & 107.92213 & 26.98450 \\
\hline $\mathrm{S} 2$ & Wild, Xinzhou town, Huangping county, Guizhou province & 107.92213 & 26.99918 \\
\hline S3 & Wangsi town, Duyun county, Guizhou province & 107.51576 & 26.30596 \\
\hline S4 & Huaxi district, Guiyang city, Guizhou province & 107.27755 & 26.73151 \\
\hline S5 & Cultivated in Meitan county, Zunyi city, Guizhou province for 2 years & 107.45728 & 28.02350 \\
\hline S6 & Cultivated in Meitan county, Zunyi city, Guizhou province for 3 years & 107.45728 & 28.02350 \\
\hline S7 & Guizhou Academy of Agricultural Sciences (Huaxi district, Guiyang city, Guizhou province) & 107.27755 & 26.73151 \\
\hline S8 & Baiduo village, Shibing county, Guizhou province for 2 years & 108.15923 & 27.14368 \\
\hline S9 & Baiduo village, Shibing county, Guizhou province for 3 years & 108.15923 & 27.14368 \\
\hline S10 & Niudachang town, Shibing county, Guizhou province for 2 years & 107.92594 & 27.14054 \\
\hline S11 & Niudachang town, Shibing county, Guizhou province for 3 years & 107.92594 & 27.14054 \\
\hline $\mathrm{S} 12$ & Wild, Shibing county, Guizhou province & 108.06787 & 27.12923 \\
\hline S13 & Changhao Chinese Medicine Development Co., Ltd. (Shibing county, Guizhou province) & 107.94637 & 26.55361 \\
\hline S14 & Zhenjiang town, Gaozhou city, Guangdong province & 110.84849 & 21.94484 \\
\hline S15 & Shigu town, Gaozhou city, Guangdong province & 110.86943 & 21.95980 \\
\hline S16 & Yangchun city, Guangdong province & 111.79154 & 22.17044 \\
\hline S17 & Luanchuan county, Luoyang city, Henan province & 111.61577 & 33.78570 \\
\hline $\mathrm{S} 18$ & Panzhihua city, Miyi county, Sichuan province & 102.11034 & 26.89069 \\
\hline
\end{tabular}

was complemented by $70 \%$ ethanol and mixed well. After standing, the supernatant was filtered through filter paper. During the process, 18 RPM samples were prepared 3 replicates. Before UPLC-QTOF/MS analysis, the filtered supernatant was filtered through a $0.22 \mu \mathrm{m}$ microporous membrane and $2 \mu \mathrm{L}$ aliquot was injected.

In addition, quality control (QC) sample was prepared by mixing $100 \mu \mathrm{L}$ supernatants of 18 geographical RPM samples to validate stability of LC-MS system. It was injected for 3 times before beginning the whole sample list to condition or balance the system. During the analytical run, QC sample was injected every 9 RPM samples to further monitor and investigate the stability and analytical variability of the system. After that, the change degree of the analytical system in the analysis process could be obtained and determined, which was critical for assessing the variation and reliability of the analytical results.

\section{UPLC-QTOF/MS conditions}

Samples were analyzed using a Waters ACQUITY UPLC I-Class system (Waters Corporation, Milford, USA). An Acquity UPLC HSS T3 C18 column (2.1 mm i.d. $\times 100 \mathrm{~mm}, 1.8 \mu \mathrm{m})$ was used for chromatographic separation. All samples were run in a random and nongrouped order. The flow phases consisted of $0.1 \%$ formic acid in water (A) and acetonitrile (B). The program of gradient elution was set as follows: $0-16 \mathrm{~min}, 5-60 \% \mathrm{~B}$; $16-20 \mathrm{~min}, 60-100 \% \mathrm{~B}$. The flow rate was $0.4 \mathrm{~mL} / \mathrm{min}$. The temperatures of column oven and auto-sampler were maintained at 35 and $10{ }^{\circ} \mathrm{C}$ during the analysis, respectively. The sample injection volume was $2 \mu \mathrm{L}$.

MS spectrometry detection was operated on a Waters Xevo G2-XS QTOF/MS (Waters, Manchester, UK) equipped with the UPLC system through an electrospray ionization (ESI) interface in negative and positive ion modes. The ESI source parameters were maintained as follows: capillary voltage $1.0 \mathrm{kV}$, cone voltage $40 \mathrm{~V}$, source temperature $110{ }^{\circ} \mathrm{C}$, desolvation temperature $450{ }^{\circ} \mathrm{C}$. Nitrogen was used as cone gas and desolvation gas with flow of 50 and $800 \mathrm{~L} / \mathrm{h}$, respectively. Argon was used as collision gases. The acquisition range of MS scanning was from $m / z 50$ to $1200 \mathrm{Da}$ in $\mathrm{MS}^{\mathrm{E}}$ continuum mode. By using a collision energy ramp from 10 to $30 \mathrm{~V}$, the MS/ MS fragment information was obtained. The mass accuracy and reproducibility of UPLC-QTOF/MS was validated by the reference lock mass of leucine-enkephalin $\left(\mathrm{ESI}^{+}: m / z\right.$ 556.2771; $\left.\mathrm{ESI}^{-}: m / z 554.2615\right)$ with the concentration of $100 \mathrm{pg} / \mu \mathrm{L}$ and the flow rate of $10 \mu \mathrm{L} / \mathrm{min}$. Data acquisition was performed using Masslynx ${ }^{\text {TM }}$ v 4.1 (Waters, Manchester, UK).

\section{Data processing}

All raw data of RPM samples in the LC-MS runs were loaded on Progenesis QI software (v2.0). By using the "assess all runs in the experiment for suitability", QC2 was automatically selected as the alignment reference. Next, the peaks of all other runs were aligned by comparison with QC2. After that, the experiment design (QC group and S1-S18 groups) was set, the peaks of all samples 
were picked and convoluted. And then, all data of the peaks were exported into the EZinfo software (v3.0) for PLS-DA analysis. The necessary data were filtered and then were imported into Progenesis QI software (v2.0) to identify the compounds by its powerful Metascope search engine in the software according to the accurate mass, isotope distribution, fragment ions, collision crosssectional area and many other parameters.

The significant differences of the markers in different RPM species were analyzed by one-way analysis of variance (ANOVA). The results are shown as mean \pm SD. The differences were considered statistically significant at $P \leq 0.05$.

\section{Results}

\section{Data analysis by Progenesis QI}

Multivariate statistical tools was used to observe all differences among the RPM samples from different geographical origins. Firstly, the 3D LC/MS data acquired by Masslynx ${ }^{\mathrm{TM}} \mathrm{v} 4.1$ were converted into a $2 \mathrm{D}$ ion intensity map as an exact mass retention time (EMRT) pair by using Progenesis QI. During the process, the RPM QC2 sample was automatically selected as the alignment reference by Progenesis QI, and all other RPM samples were aligned with $\mathrm{QC} 2$ as the reference. The representative peak alignment results and chromatograms between QC2 and S14b were shown in Fig. 1. Figure 1a was a vector alignment window, there were 414 vectors; Fig. 1b, c both were 2D ion intensity map. Figure $1 \mathrm{c}$ also showed the matching results for peak alignment between QC2 and S9b, the score was $96.1 \%$. The matching score range between QC2 and other RPM sample was from 90.3\% (S15c) to $97.9 \%$ (S18b). The ordinate of Fig. 1a-c represented the retention time (Rt), and the abscissa of them was $\mathrm{m} / z$. Figure $1 \mathrm{~d}$ was the total ion chromatograms (TIC), green and purple chromatograms represented QC2 and S9b, representatively.

Then, the experiment was designed, 60 samples were divided into 19 groups, including QC and S1-S18 groups. Next, all peaks of RPM samples were picked and convoluted. The parameters of sensitivity value was set at 3 and the minimum peak width was set at $0.15 \mathrm{~min}$, respectively. Under the condition, the best balance could be obtained with the most true feature ion signals and the least random noise. Total 24,530 peaks were observed in the $2 \mathrm{D}$ ion intensity map, which was shown in Additional file 2: Figure S1. The normalization graphs for the RPM samples are shown in Additional file 3: Figure S2.
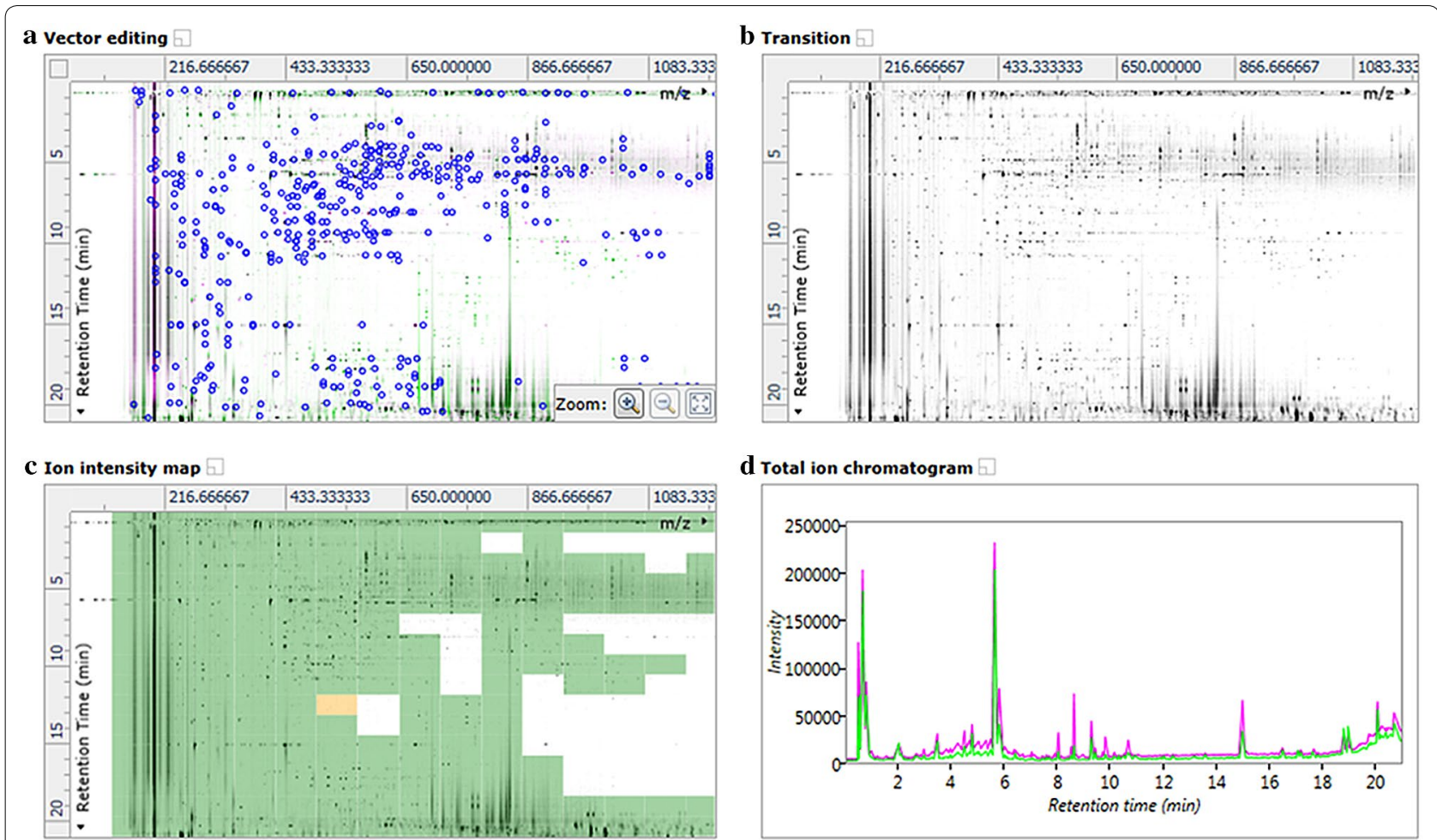

d Total ion chromatogram

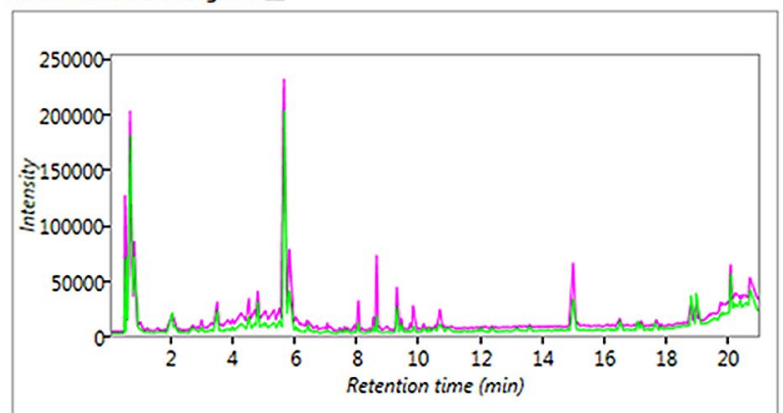

Alignment quality: $\square$ Good $\square$ OK $\square$ Needs review

Fig. 1 The translation process by Progenesis QI software (a vector alignment window; $\mathbf{b}$ and $\mathbf{c} 2 \mathrm{D}$ ion intensity maps; $\mathbf{d}$ total ion chromatograms, green: QC2; purple: S9b) 


\section{PLS-DA analysis for RPM samples}

After that, all data were exported into the EZinfo software (v3.0) for PLS-DA analysis. The outliers and classification trends among the 18 kinds of RPM samples could be observed in PLS-DA results (Fig. 2). In the score plot obtained by PLS-DA, there was a clear differentiation between RPM S1-S12 groups and S13 group, indicating that RPM sample from Changhao Chinese Medicine Development Co., Ltd. was very different from other samples in Guizhou province. RPM samples from Guangdong province (S14-S16 groups) clustered together and separated from Guizhou samples. RPM samples from Henan (S17 group) and Sichuan
(S18 group) provinces were closer to Guizhou samples, and located father from Guangdong samples. $R^{2} Y$ and $Q^{2}$ of the PLS-DA model were 0.771 and 0.634 , respectively, which suggested that the PLS-DA model had good adaptability and predictability. Among the RPM samples from the same place of Guizhou province, the samples between S1 and S2 clustered together respectively, indicating that cultivated and wild RPM samples in Xinzhou town had significant difference; the separation between S5 and S6, S8 and S9, S10 and S11 indicated that cultivated RPM samples for 2 and 3 years in Meitan county, Baiduo village, and Niudachang town also had significant difference.

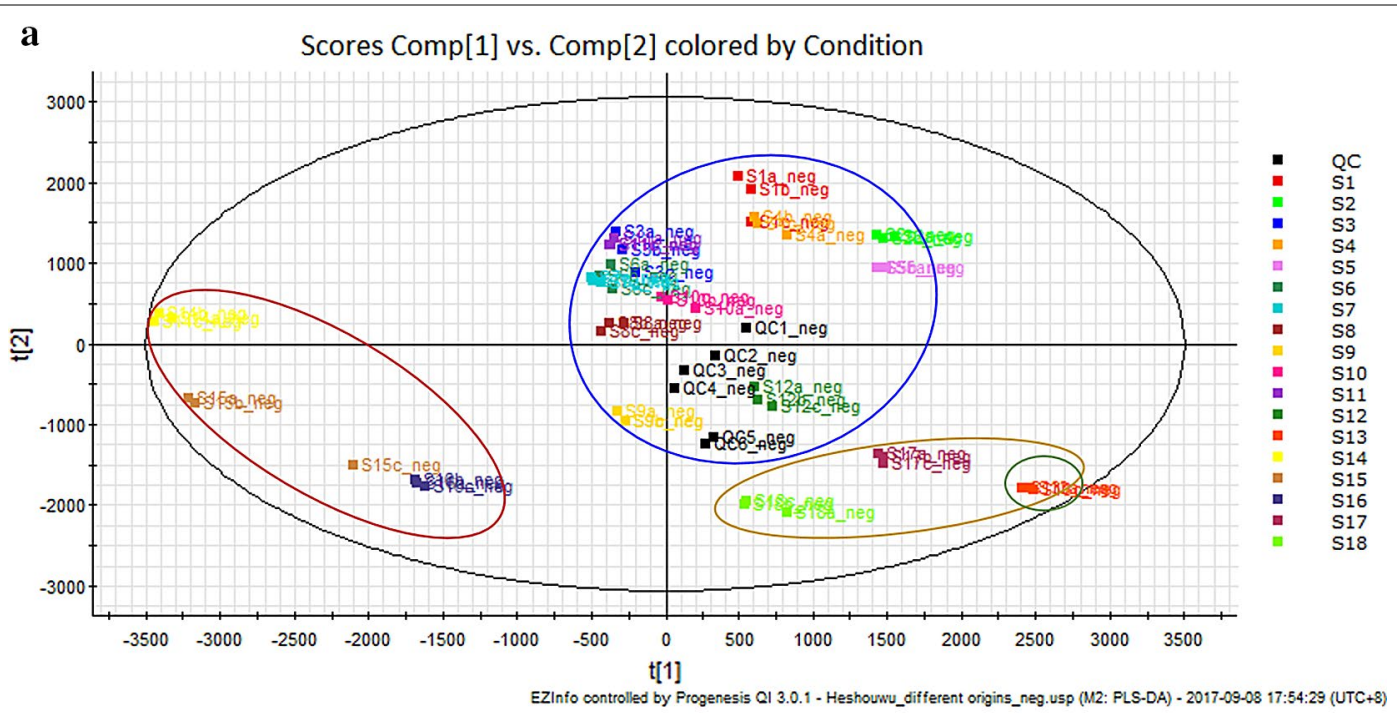

b

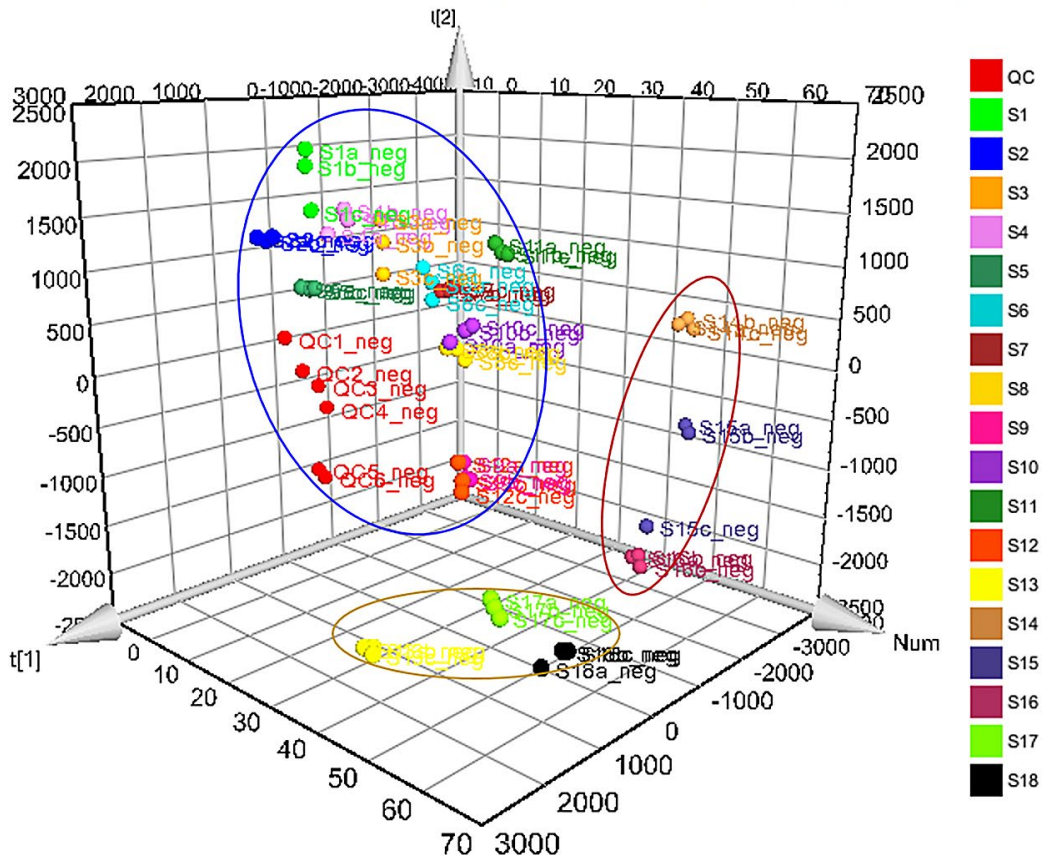

Fig. 2 The PLS-DA 2D score plot (a) and 3-D score plot (b) of RPM samples from different geographical origins 
Identification of chemical markers

Identification of potential chemical markers in RPM samples from different geographical origins was carried out on basis of the retention behavior and mass assignment using Progenesis QI software. First, PLS-DA model was constructed from the EZinfo software. From loading plots (Fig. 3a) and VIP plots (Fig. 3b) of that model, the interested potential biomarkers could be extracted.
Additionally, an ANOVA $\mathrm{P} \leq 0.05$, a maximum fold change $\geq 2$ and VIP value $>1$ were set as the restriction conditions to select the significant changing compounds and reduce the "false discovery rate (FDR)". Next, the Progenesis MetaScope, ChemSpider (http://www. chemspider.com/) and Element composition methods of Progenesis QI software was used for preliminary determination of the chemical markers. The mass tolerance



Fig. 3 The PLS-DA loading plot (a) and VIP plot (b) of RPM samples from different geographical origins 
between the measured $m / z$ values and exact mass of the interest compounds, and the relative mass error of the performed theoretical fragmentation both were set to within $5 \mathrm{ppm}$. Then, under the targeted MS/MS mode, the MS/MS spectrum of chemical markers was obtained. Finally, some chemical markers were identified by comparison with the standard reference; and others were identified by MS/MS spectrum, online database, element composition results, and literatures.

According to the protocol detailed above, 9 chemical markers (C1-C9) in RPM samples from different geographical origins were identified (Table 2). Among them, 4 chemical markers including $\mathbf{C 1}, \mathbf{C} 3, \mathbf{C} 4$ and C9 were identified by comparing with their reference compounds. Other 5 compounds were tentatively identified on basis of their molecular ion information and fragments generated by precursor ions. Herein, the $\mathbf{C 5}$ with $\mathrm{Rt}-m / z$ of $8.53-407.1347$ in negative ion mode was detailed as an example to illustrate the identification process. Firstly, the accurate mass of the marker
$\left([\mathrm{M}-\mathrm{H}]^{-}\right.$at $m / z$ 407.1347) was found from the mass spectrum (Fig. 4). Secondly, specific MS/MS information about fragmentation pattern of the marker was acquired from QTOF system. The main fragment ions of the marker in the negative ion spectrum were observed at $\mathrm{m} / \mathrm{z} 245.0819,230.0948,202.0635$, and 159.0451, which could be the $[\mathrm{M}-\mathrm{H}]^{-}$of lost $-\mathrm{C}_{6} \mathrm{H}_{12} \mathrm{O}_{5}$, $\mathrm{C}_{6} \mathrm{H}_{12} \mathrm{O}_{6},-\mathrm{C}_{8} \mathrm{H}_{14} \mathrm{O}_{5},-\mathrm{C}_{10} \mathrm{H}_{19} \mathrm{O}_{6}$, respectively. $\mathrm{C}_{20} \mathrm{H}_{24} \mathrm{O}_{9}$ was located as the candidate due to its high mass accuracy among the possible compounds. Finally, the chemical compound was identified as torachrysone-8-O-glucoside (C5) according to the ChemSpider database and literature $[20,21]$.

The data of 6 replicates of QC sample were analyzed to evaluate the repeatability of LC-MS method. The relative standard deviations (RSD\%) of peak areas, Rt and $m / z$ were $5.73-13.42,0-0.25$ and $0.00012-0.00301 \%$, respectively. QC sample maintained in auto sampler at $4{ }^{\circ} \mathrm{C}$ for $4,8,12,24,28,32 \mathrm{~h}$ were tested to assess the post-preparation stability of samples. The relative errors of peak

Table 2 Chemical markers in RPM samples from different geographical origins by UPLC-QTOF/MS

\begin{tabular}{|c|c|c|c|c|c|c|c|c|}
\hline No. & $\begin{array}{l}t \\
(\min )\end{array}$ & $m / z(-)$ & Fragment information & Compound name & Compound type & Formula & Max fold change & VIP value \\
\hline C1 & 0.79 & 191.0197 & $191.0197,173.0091$ & Citric acid & Organic acids & $\mathrm{C}_{6} \mathrm{H}_{8} \mathrm{O}_{7}$ & 4.72 & 4.11 \\
\hline C2 & 2.86 & 881.1917 & $\begin{array}{l}\text { 881.1917, 863.1829, } \\
\text { 591.1144, 217.0506, } \\
152.0015\end{array}$ & $\begin{array}{l}\text { 3,3'-di-O-Galloyl-procya- } \\
\text { nidin B2 }\end{array}$ & Polyphenols & $\mathrm{C}_{44} \mathrm{H}_{34} \mathrm{O}_{20}$ & 8.12 & 5.12 \\
\hline C3 & 5.68 & 405.2810 & $\begin{array}{c}405.2810,243.1911 \\
227.0425,215.0702 \\
201.0545,173.0596\end{array}$ & $\begin{array}{l}\text { 2,3,5,4'-Tetrahydroxystil- } \\
\text { bene-2-O-glucoside }\end{array}$ & Stilbene glycosides & $\mathrm{C}_{20} \mathrm{H}_{22} \mathrm{O}_{9}$ & 2.01 & 6.15 \\
\hline C4 & 8.06 & 431.0981 & $\begin{array}{c}431.0981,269.0455 \\
265.0506,263.0350 \\
253.0506,241.0506 \\
227.0350,224.0478 \\
210.0322,182.0373\end{array}$ & Emodin-8-O-glucoside & $\begin{array}{l}\text { Anthraquinone glyco- } \\
\text { sides }\end{array}$ & $\mathrm{C}_{21} \mathrm{H}_{20} \mathrm{O}_{10}$ & 31.99 & 5.20 \\
\hline C5 & 8.53 & 407.1347 & $\begin{array}{l}407.1347,245.0819 \\
230.0948,202.0635 \\
159.0451\end{array}$ & $\begin{array}{l}\text { Torachrysone-8-O- } \\
\text { glucoside }\end{array}$ & $\begin{array}{l}\text { Anthraquinone glyco- } \\
\text { sides }\end{array}$ & $\mathrm{C}_{20} \mathrm{H}_{24} \mathrm{O}_{9}$ & 7.78 & 4.28 \\
\hline C6 & 8.65 & 473.1085 & $\begin{array}{l}473.1085,456.1062 \\
431.0983,269.0455 \\
265.0506,253.0506 \\
240.0428,227.0350 \\
224.0478,210.0322 \\
182.0373\end{array}$ & $\begin{array}{l}\text { Emodin-O-(acetyl)- } \\
\text { hexoside }\end{array}$ & $\begin{array}{l}\text { Anthraquinone deriva- } \\
\text { tives }\end{array}$ & $\mathrm{C}_{23} \mathrm{H}_{22} \mathrm{O}_{11}$ & 4.42 & 7.54 \\
\hline C7 & 9.30 & 487.0638 & $\begin{array}{l}487.0638,470.0734 \\
445.0369,283.0604 \\
240.0428,212.0478 \\
197.0244,184.0505 \\
169.0278\end{array}$ & $\begin{array}{l}\text { Physcion-O-(acetyl)- } \\
\text { hexoside }\end{array}$ & $\begin{array}{l}\text { Anthraquinone deriva- } \\
\text { tives }\end{array}$ & $\mathrm{C}_{23} \mathrm{H}_{22} \mathrm{O}_{12}$ & 4.73 & 3.80 \\
\hline C8 & 10.18 & 285.0398 & $\begin{array}{r}285.0398,269.0455 \\
253.0506,240.0428 \\
227.0350,224.0478 \\
210.0322,182.0373\end{array}$ & Citreorosein & Anthraquinones & $\mathrm{C}_{15} \mathrm{H}_{10} \mathrm{O}_{6}$ & 34.91 & 4.25 \\
\hline C9 & 12.50 & 283.0604 & $\begin{array}{c}283.0604,240.0428 \\
212.0478,197.0244 \\
184.0505,169.0278\end{array}$ & Physcion & Anthraquinones & $\mathrm{C}_{16} \mathrm{H}_{12} \mathrm{O}_{5}$ & 2567.78 & 6.46 \\
\hline
\end{tabular}




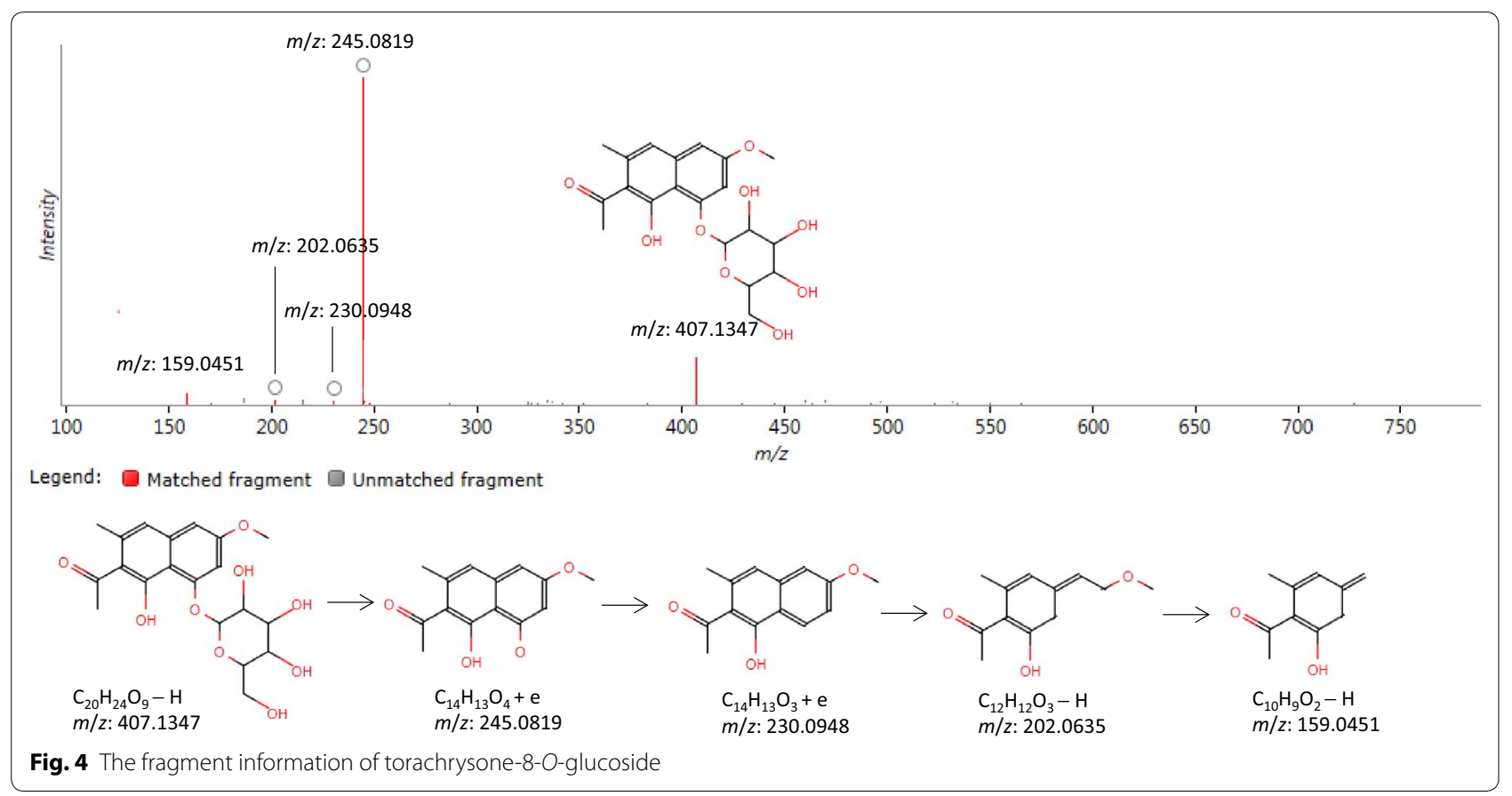

areas were $<13.42 \%$ demonstrating good repeatability and stability of the method.

Furthermore, in order to characterize the differences more clearly, the relative intensity of chemical markers in RPM samples from different geographical origins was shown in Table 3 and Fig. 5. The max fold change of C1-C9 were 4.72, 8.12, 2.01, 31.99, 7.78, 4.42, 4.73, 34.91 and 2567.78, respectively. And the results of Fig. 5C9 and Table 3 showed that the content of C9 in 18 RPM samples had the most different, and the content of $\mathbf{C 9}$ in S18 was the highest, which was about 4 time than S13; the content of $\mathbf{C 9}$ in S2 and S10 were similar, which was lower than S13 and S18, but higher than other 15 RPM samples; the content of C9 in S1, S3-S9, S11, S12, S14S17 were similar. The content trend of C4 (Fig. 5C4) in 18 RPM samples was similar with C9. The content of C8 (Fig. 5C8) in S9 was the highest; the content of S1, S3, S13, and S16 were lower than S9, but higher than S2, S4-S8, S10-S12, S14, S15, S17 and S18. The content of S4 and $\mathrm{S} 7$ was similar, which was higher than other 16 RPM samples. The content of C5 (Fig. 5C5) and C7 (Fig. 5C7) in S5 was the highest, the content of them in S9 was the lowest, but the content of $\mathbf{C 7}$ in $\mathrm{S} 9$ was similar with that in S9. The content of $\mathbf{C 1}$ (Fig. 5C1) and $\mathbf{C 3}$ (Fig. 5C3) in S9 and S14 were the highest, respectively; but the content of them in $\mathrm{S} 13$ both were the lowest. The content of $\mathbf{C 2}$ (Fig. 5C2) and C6 (Fig. 5C6) in S4 were the highest, but the content of $\mathbf{C 2}$ in $\mathrm{S} 7$ was similar with $\mathrm{S} 4$; the content of them in S16 both were the lowest, but the content of C2 (Fig. 5C2) in S13 and S15 was almost equal to that of
S16; and the content of C6 (Fig. 5C6) in S14 was almost equal to the content in S16.

\section{Discussion}

Because of the clinical benefits, TCM is becoming more and more attractive around the world. Therefore, it is an important issue to carry out the quality control study of TCM for its application and development. To control the quality of Chinese medicine and its products, studying the source of TCMs is a key. In the present study, 18 RPM samples were collected from 10 counties, 4 provinces of China. And 9 representative chemical markers related to the differences among the 18 RPM samples were identified. The content of $\mathbf{C 1}$ and $\mathbf{C 8}$ in S9 were the highest; while the content of $\mathbf{C 5}$ and $\mathbf{C 7}$ in S9 were the lowest. The content of C1, C2 and C3 in S13 and S18 were the lowest. The content of $\mathbf{C 2}$ and $\mathbf{C 6}$ were the highest. The content of C3 in S14 was the highest. The content of C4 and $\mathbf{C 9}$ in S18 were the highest. The content of C5 and C7 in S5 were the highest. The results suggested that $\mathbf{C 1}$, C5 and C7 could be used as specific chemical markers for S9 and S5; C1, C2 and C3 could be used as special chemical markers for S13; C1, C2, C3, C4 and C9 could be used as specific chemical markers for S18; C3 could be used as unique chemical markers for S14. The PLS-DA results of different RPM samples showed that the RPM from Guizhou provinces were different from the RPMs of Guangdong, Henan and Sichuan provinces, indicating that RPM samples from Guizhou province had some similarity. The samples from Guangdong, Henan and 


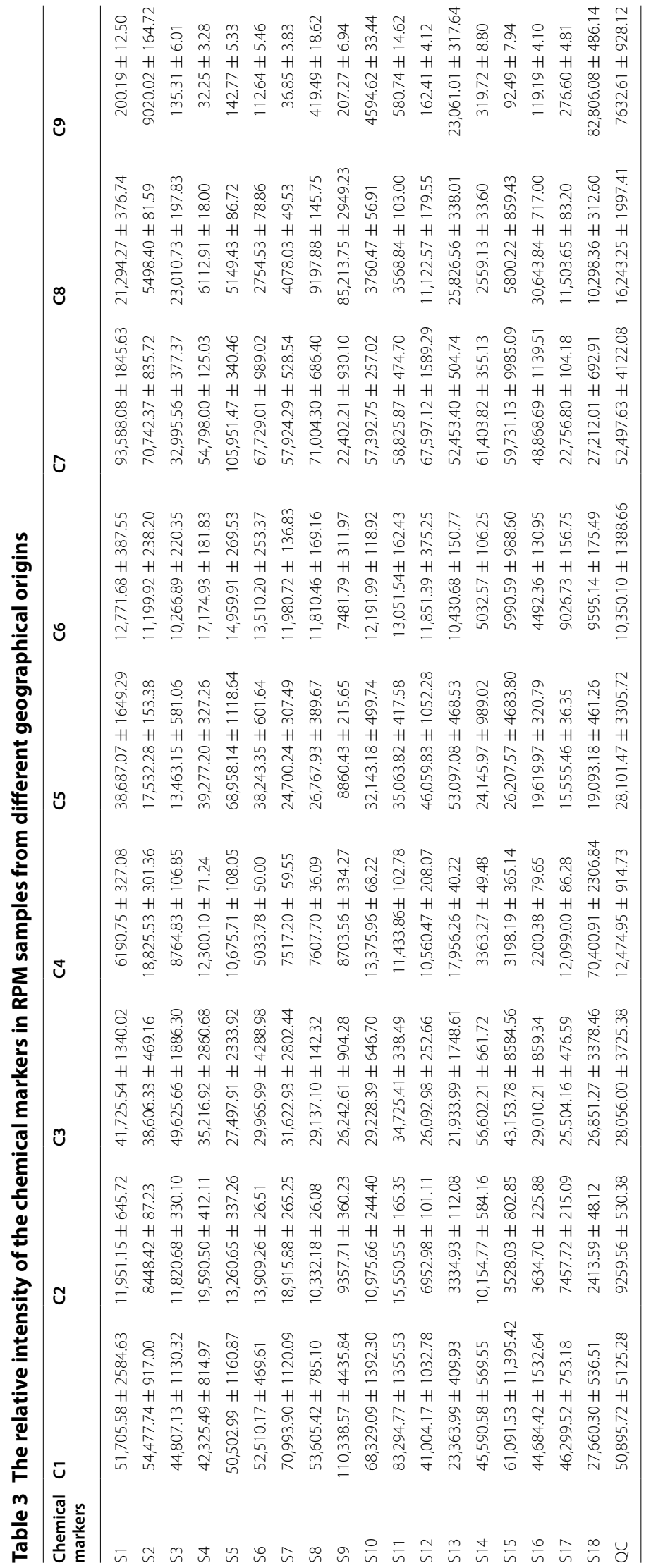




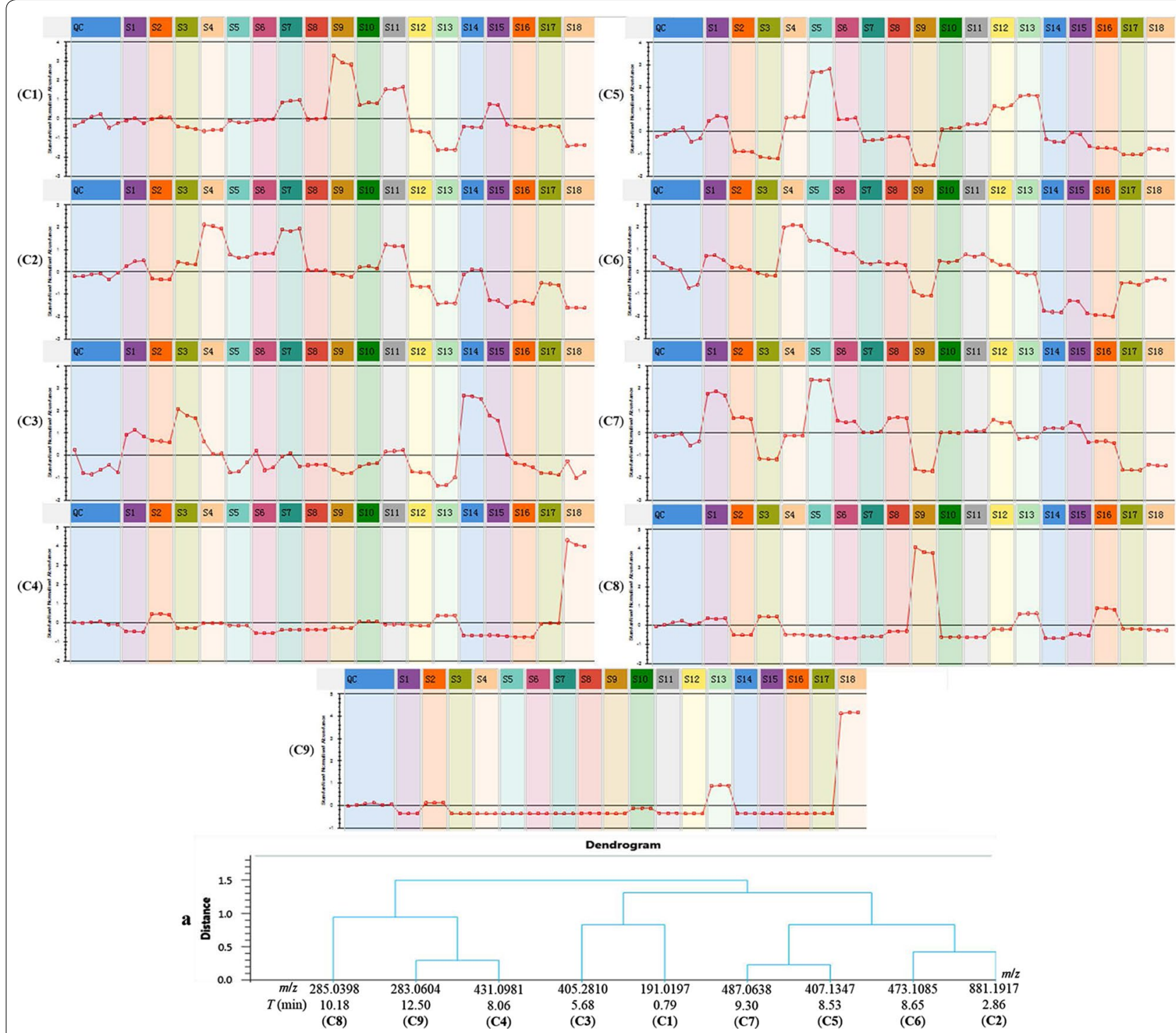

Fig. 5 The standard normalized abundance of 9 chemical markers $(\mathbf{C 1}-\mathbf{C 9})$ and the dendrogram (a) of 9 chemical markers

Sichuan provinces were also clustered together, respectively. And there were significant difference between the RPM sample for 2 and 3 years.

RPM contains a variety of anthraquinones, stilbene glycosides, phospholipids, phenols, flavonoids and tannins. Stilbene glycosides are a class of natural ingredients with a variety of physiological activities. The most active components including aloe-emodine, emodin, rhein and physcione were identified as the antioxidant anthraquinones [22]. The anthraquinone glycoside from RPM could significantly accelerate $\mathrm{T}$ and $\mathrm{B}$ lymphocytes proliferation in vitro, improve macrophages phagocytosis, increase TNF secretion activity and activity of NK cells, accelerate mixed lymphocyte reaction, and antagonize restraining effect of lymphocyte proliferation by mitomycin [23].
RPM samples from 18 different geographical origins were discriminated using UPLC-QTOF/MS coupled with chemometrics method in the present study. Those chemical markers with significant pharmacological activities could be used to distinguish the geographical origins of RPM samples. The results will help to develop strategies for protection and utilization of RPM samples. Chemometrics technique has potential to be used for discovering active components and evaluating the therapeutic effect and toxicity of TCMs, related to the complex composition and different growth geographical environment, and help us to find the natural substitute for the geographical origins herb medicine depleting more rapidly. 


\section{Conclusion}

Classification and distinction of TCMs based on geographical origins are important basis for ensuring their quality and safety. The chemical constitution of RMP samples from different areas were characterized by UPLC-QTOF/MS method. PLS-DA method was applied to classify the samples from different origins. And the marker compounds contributing to the differentiation of RPMs were observed and identified. Nine chemical markers were tentatively identified and semi-quantified in different geographical RPM samples. The individual peak data of those chemical markers with significant content difference in different RPM regions were calculated. Those chemical markers could be applied to distinguish the geographical origins of different geographical RPM samples. The results showed that chemometric technique has potential to be used for discovering active components of TCMs, related to the complex composition and different growth geographical environment, and help us to find the natural substitute for the geographical origins herb medicine depleting more rapidly.

\section{Additional files}

Additional file 1. The Minimum Standards of Reporting Checklist.

Additional file 2: Figure S1. The peak picking-ion map.

Additional file 3: Figure S2. The normalization graphs of RPM samples from different geographical origins.

\section{Abbreviations}

RPM: Radix Polygoni Multiflori; UPLC-QTOF/MS: ultra-high performance liquid chromatography quadrupole time of flight mass spectrometry; PLS-DA: partial least squared discriminant analysis; TCM: traditional Chinese medicines; TLC: thin-layer chromatography; HPLC: high-performance liquid chromatography; IR: infrared spectrum; ICP-AES: inductively coupled plasma-atomic emission spectrometer; MS: mass spectrometry; DMPK: drug metabolism and pharmacokinetics; QC: quality control; EMRT: exact mass retention time; TIC: total ion chromatograms; ANOVA: analysis of variance; FDR: false discovery rate.

\section{Authors' contributions}

JFT and WXL contributed equally to this work. JFT and XLL conceived and designed the study. JFT and YHL collected RPM samples from different geographical areas. WXL and YJC performed the LC-MS analysis experiment and collected the data. FZ and YZ prepared the RPM samples. WXL wrote the manuscript. ZJM involved in the initial experimental design, and provided a great help and careful guide for the twice modification of the manuscript including the data processing analysis and grammar part. All authors read and approved the final manuscript.

\section{Acknowledgements}

We are pleased to thank Waters China Ltd. for technical support.

\section{Competing interests}

The authors declare that they have no competing interests.

\section{Availability of data and materials}

All data generated or analyzed during this study are included in this published article.

\section{Consent for publication}

All of authors consent to publication of this study in journal of Chinese Medicine.

\section{Ethics approval and consent to participate}

Not applicable.

\section{Funding}

This work was supported by 2015 Special subject of Chinese medicine research in Henan Province (No. 2015ZY02101).

\section{Publisher's Note}

Springer Nature remains neutral with regard to jurisdictional claims in published maps and institutional affiliations.

Received: 12 July 2017 Accepted: 1 December 2017

Published online: 08 December 2017

\section{References}

1. Man SC, Chan KW, Lu JH, Durairajan SS, Liu LF, Li M. Systematic review on the efficacy and safety of herbal medicines for vascular dementia. Evid Based Complement Altern Med. 2012. https://doi. org/10.1155/2012/426215.

2. He Y, Wang F, Chen SQ, Liu M, Pan W, Li X. The protective effect of Radix Polygoni Multiflori on diabetic encephalopathy via regulating myosin light chain kinase expression. J Diabetes Res. 2015. https://doi. org/10.1155/2015/484721.

3. Man SC, Chan KW, Lu JH, Durairajan SS, Liu LF, Li M. Systematic review on the efficacy and safety of herbal medicines for vascular dementia. Evid Based Complement Altern Med. 2012. https://doi. org/10.1155/2012/426215.

4. He Y, Wang F, Chen SQ, Liu M, Pan W, Li X. The protective effect of Radix Polygoni Multiflori on diabetic encephalopathy via regulating myosin light chain kinase expression. J Diabetes Res. 2015. https://doi. org/10.1155/2015/484721.

5. He Y, Wang F, Chen SQ, Liu M, Pan W, Li X. The protective effect of Radix Polygoni Multiflori on diabetic encephalopathy via regulating myosin light chain kinase expression. J Diabetes Res. 2015. https://doi. org/10.1155/2015/484721.

6. Hong CY, Lo YC, Tan FC, Wei YH, Chen CF. Astragalus membranaceus and Polygonum multijlorum protect rat heart mitochondria against lipid peroxidation. Am J Chin Med. 1994;22:63-70.

7. Jung KA, Min HJ, Yoo SS, Kim HJ, Choi SN, Ha CY, Kim HJ, Kim TH, Jung WT, Lee OJ, Lee JS, Shim SG. Drug-induced liver injury: twenty five cases of acute hepatitis following ingestion of Polygonum multiflorum Thunb. Gut Liver. 2011:5:493-9.

8. Chinese Pharmacopoeia Commission. Pharmacopoeia of the People's Republic of China. Beijing: China Medical Science Press; 2005. p. 122-3.

9. Chinese Pharmacopoeia Commission. Pharmacopoeia of the People's Republic of China. Beijing: China Medical Science Press; 2010. p. 164-5.

10. Chen Y, Jiang B, Zeng YE. Study on the geographic distribution and ecological environmental characteristics of Polygonum multiflorum based on GIS. China Pharm. 2011:22:3726-8.

11. Gao XX, Yan HJ, Liang CQ, Chen XY. Preliminary study on TLC fingerprint of Radix Polygoni Multiflori from different areas. J Chin Med Mater. 2007;30:407-9.

12. Li SF, Zheng CZ, Zhang L, Lan CW, He DX, Yao WF, Shang EX, Ding AW. HPLC fingerprint on roots of Polygonum multiflorum from different habitats. Chin Trade Herbal Drugs. 2015;46:2149-54.

13. Liang ZT, Leung NN, Chen HB, Zhao ZZ. Quality evaluation of various commercial specifications of Polygoni Multiflori Radix and its dregs by determination of active compounds. Chem Cent J. 2012;6:53.

14. Yuan YF, Tao ZH, Tian CH, Liu JX, Huang SS. Assessment of Polygonum multiflorum based on different origins by infrared spectra and chemometrics. Lishizhen Med Mater Med Res. 2011;22:1835-7. 
15. Yan HJ, Fang ZJ. Study on determination and principal component analysis of inorganic elements in Polygonum multiflorum from different areas. China J Chin Mater Med. 2008;33:416-9.

16. Zhu ZW, Li J, Gao XM, Amponsem E, Kang LY, Hu LM, Chang YX. Simultaneous determination of stilbenes, phenolic acids, flavonoids and anthraquinones in Radix Polygoni Multiflori by LC-MS/MS. J Pharm Biomed Anal. 2012;62:162-6.

17. Liang ZT, Leung NN, Chen HB, Zhao ZZ. Quality evaluation of various commercial specifications of Polygoni Multiflori Radix and its dregs by determination of active compounds. Chem Cent J. 2012;6:1-7.

18. Chen ML, Chang WQ, Zhou JL, Yin YH, Xia WR, Liu JQ, Liu LF, Xin GZ. Comparison of three officinal species of Callicarpa based on a biochemome profiling strategy with UHPLC-IT-MS and chemometrics analysis. J Pharm Biomed Anal. 2017;145:666-74.

19. Liu S, Liang YZ, Liu HT. Chemometrics applied to quality control and metabolomics for traditional Chinese medicines. J Chromatogr B Anal Technol Biomed Life Sci. 2016;1015-1016:82-91.
20. Lin $L F$, Lin HM, Zhang M, Ni BR, Yin $X B, Q u C H, N i$ J. A novel method to analyze hepatotoxic components in Polygonum multiflorum using ultraperformance liquid chromatography-quadrupole time-of-flight mass spectrometry. J Hazard Mater. 2015;299:249-59.

21. Qiu XH, Zhang J, Huang ZH, Zhu DY, Xu W. Profiling of phenolic constituents in Polygonum multiflorum Thunb. by combination of ultrahigh-pressure liquid chromatography with linear ion trap-orbitrap mass spectrometry. J Chromatogr A. 2013;1292:121-31.

22. Zuo Y, Wang C, Lin Y, Guo J. Simultaneous determination of anthraquinones in Radix Polygoni Multiflori by capillary gas chromatography coupled with flame ionization and mass spectrometric detection. J Chromatogr A. 2008;1200:43-8.

23. Sun GB, Guo BJ, Li XE, Huang JN, Xue HB, Sun XB. The effect of anthraquinone glycoside from Polygonum multiflorum Thunb. on cellular immunological function in mice. Pharmacol Clin Chin Mater Medica. 2006:22:30-2

\section{Submit your next manuscript to BioMed Central and we will help you at every step:}

- We accept pre-submission inquiries

- Our selector tool helps you to find the most relevant journal

- We provide round the clock customer support

- Convenient online submission

- Thorough peer review

- Inclusion in PubMed and all major indexing services

- Maximum visibility for your research

Submit your manuscript at www.biomedcentral.com/submit 